This item was submitted to Loughborough's Research Repository by the author.

Items in Figshare are protected by copyright, with all rights reserved, unless otherwise indicated.

\title{
Automatic segmentation of adipose tissue from thigh magnetic resonance images
}

PLEASE CITE THE PUBLISHED VERSION

http://dx.doi.org/10.1007/978-3-642-39094-4_51

\section{VERSION}

AM (Accepted Manuscript)

\section{PUBLISHER STATEMENT}

This work is made available according to the conditions of the Creative Commons Attribution-NonCommercialNoDerivatives 4.0 International (CC BY-NC-ND 4.0) licence. Full details of this licence are available at: https://creativecommons.org/licenses/by-nc-nd/4.0/

\section{LICENCE}

CC BY-NC-ND 4.0

\section{REPOSITORY RECORD}

Purushwalkam, Senthil, Baihua Li, Qinggang Meng, and Jamie S. McPhee. 2019. "Automatic Segmentation of Adipose Tissue from Thigh Magnetic Resonance Images”. figshare. https://hdl.handle.net/2134/20258. 


\title{
Automatic Segmentation of Adipose Tissue from Thigh Magnetic Resonance Images
}

\author{
Senthil Purushwalkam ${ }^{1}$, Baihua $\mathrm{Li}^{2}{ }^{\star}$, Qinggang Meng ${ }^{3}$, and Jamie McPhee ${ }^{4}$ \\ 1 Electronics and Electrical Dept., Indian Institute of Technology Guwahati, India \\ 2 School of Computing, Mathematics and Digital Technology, Manchester \\ Metropolitan University, UK \\ 3 Dept. of Computer Science, Loughborough University, UK \\ 4 School of Healthcare Science, Manchester Metropolitan University, UK
}

\begin{abstract}
Automatic segmentation of adipose tissue in thigh magnetic resonance imaging (MRI) scans is challenging and rarely reported in the literature. To address this problem, we propose a fully automated unsupervised segmentation method involving the use of spatial intensity constraints to guide the segmentation process. The novelty of this method lies in two aspects: firstly, an adaptive distance classifier, incorporating intra-slice spatial continuity, is used for robust region growing and segmentation estimation; secondly, polynomial based intensity inhomogeneity maps are generated to model inter- and intra-slice intensity variation of each pixel class and thus refine the initial classification. Our experimental results have demonstrated the effectiveness of imposing 3D intensity constraints to successfully classify the adipose tissue from muscles in the presence of image noise and considerable amounts of non-uniform MRI intensity.
\end{abstract}

Keywords: Adipose tissue, thigh MRI, intensity inhomogeneity

\section{Introduction}

During older age, humans tend to increase adipose tissue (fat mass) and decrease muscle mass and this can impact on strength, mobility and quality of life. To quantify these changes during the development of ageing or some diseases, more advanced techniques, such as magnetic resonance imaging (MRI), are required. MRI displays digitised images of different tissue types, such as connective, neural, adipose, skeletal and muscle tissue. They pose various challenges to standard image analysis techniques designed to distinguish between and quantify the contents of different tissue types [1]. This includes random image noise, spectral artefacts and tissue contrast differentiation among trials and subjects. Apart from these uncertainties, anatomically irrelevant intensity variation induced by non-uniform radio-frequency fields $(\mathrm{RF})$ introduces further complications for automatic MRI segmentation. Existing image processing software such as Osyrix,

\footnotetext{
* Corresponding author: b.li@mmu.ac.uk
} 
ImageJ and ITK-SNAP [2] do not possess the capability of automatic segmentation of adipose tissue in MRI scans of the thigh. Such tools can be principally used to semi-automatically analyse or manually draw around regions of interest to estimate areas and distances. They could work well on one or several connected slices if the region is well defined. However, the tracing results may not be propagated correctly throughout the scan when regions of interest are of irregular size, shape or suffer from various image noise. It is usually required to retrace regions of interest many times in a scan which is very laborious and prone to errors [3]. Thus, an automated procedure must be used instead.

To tackle the problem of intensity inhomogeneity, Chuang et. al. proposed the use of fuzzy C-means clustering with spatial connectivity constraints to guide segmentation [4]. This method worked under the assumptions that the pixels of each class are well connected, and image inhomogeneity is minimal. While their method works well for brain MRI, it is unlikely to work effectively to measure adipose tissue infiltration in the thigh that is irregularly distributed and disconnected, and further affected by RF non-uniformity. Parametric functions have been reported as a particular category for inhomogeneity correction [5]. Intensity inhomogeneity is usually mathematically modelled as additive or multiplicative factors. A main objective of such an approach is that the parameters need to be determined by fitting the function using a sufficient number of classified sample pixels. To obtain decent samples, manual selection and segmentation has been largely involved in many works, which were trivial and less accurate [6]. The literature is quite sparse on automatic methods to select training pixels [7].

To address the problem of intensity inhomogeneity while simultaneously removing the requirement of any user interaction, we present a hybrid unsupervised method. In contrast to other parametric modelling of inhomogeneity in a $2 D$ MRI slice, we introduce a $3 D$ polynomial-based intensity inhomogeneity map which is capable of modelling both intra- and inter-intensity variation presented in $3 \mathrm{D}$ volume of a MRI scan sequence. Taking account of spatial connectivity, we introduce an adaptive distance classifier for robust region growing and to effectively handle image noise. Combining the advantages of thresholding and the robust region growing, a sufficient amount of reliable sample pixels can be automatically generated for each class and across all MRI slices. The 3D inhomogeneity map of each class can then be calculated by polynomial fitting using these identified sample pixels. Experimental results show that the 3D intensity inhomogeneity map ultimately simplifies the classification task to a low-cost distance measurement problem, and improves the accuracy in noisy MRI. To our knowledge, this is the first report on fully automated adipose detection from thigh MRI in the presence of significant intensity non-uniformity and in the absence of ground truth for training guide.

\section{Methodology}

In our study of ageing, a 0.2 Tesla MRI scanner was used to generate high quality images of the mid-section of the thigh of one leg. These scans produced a total of 


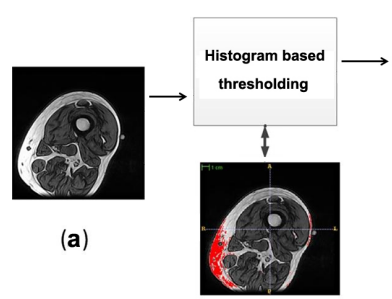

(b)

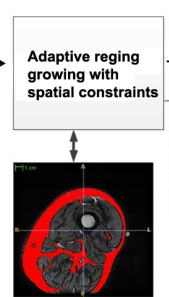

(c)

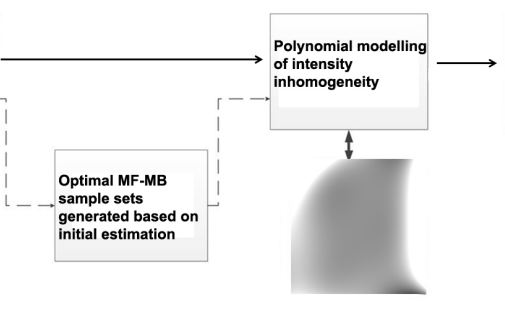

(d)

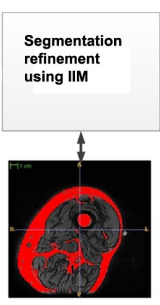

(e)

Fig. 1. Adipose detection from thigh MRI using 3D intensity inhomogeneity maps: (a) one slice in a MRI scan acquired from an elderly person; (b)histogram-based thresholding image; (c) robust region growing using adaptive distance classifier; (d)intensity inhomogeneity map; (e) segmentation result on MF.

24 serial cross-sectional slices, each representing $6.3 \mathrm{~mm}$ of thigh, with no interslice gap. Scans were performed using a Turbo 3D T1-weighted protocol. Within each slice the muscle, bone, marrow and fat need to be determined and within the series of slices the special distribution of these tissues need to be tracked for medical or research purposes.

We observe that marrow and fat have a similar high intensity value, which is well contrasted against the low intensity of muscle and bone. In this paper, we report a solution to a crucial step in fat segmentation, namely, robustly classifying thigh MRI into two pixel classes: Marrow and Fat (MF), and Muscle and Bone (MB). Based on this classification, the strongly contrasting bone can be easily distinguished from muscle, and marrow can be identified from the MF, as it is well encompassed by the bone. The proposed method includes three main stages: 1) initial MF-MB estimation (Section 2.1);2) generation of a 3D intensity inhomogeneity map (IIM) for each class (Section 2.2); and 3) refined classification based on intensity maps (Section 2.3). The overall flowchart of the algorithm is shown in Fig. 1.

\subsection{Initial MF-MB estimation}

The initial MF-MB estimation aims to automatically produce a sufficient number of reliable MF and MB sample pixels. These samples are used to calculate the 3D inhomogeneity map of each class. To this end, we begin by using a histogram based method to obtain a few high confidence MF pixels in each slice; these pixels are then used as seeds for robust region growing via an adaptive distance classifier to obtain regions of the MF class, and therefore estimation of MB pixels within the thigh area.

\section{Step 1: histogram-based thresholding}

The thigh MRIs used in our project are in 12bit grayscale, possessing intensity values in the range $[0,4095]$. A histogram is created for each slice, and the intensity distribution is equally blocked into 16 bins, each composed of 256 values. The first bin mainly contains background pixels, so we subtract these pixels 
from the image to obtain an approximation of thigh area. We then count down $2 \%$ of the remaining pixels, starting from the highest intensity pixels in the histogram. The average intensity value of the bin reached by the counting is used as MF threshold. This method succeeds in giving a number of high confidence MF samples, while rigorously avoiding any false-positive estimations.

Step 2: robust region growing using adaptive distance classifier constrained by spatial continuity

In order to obtain a good number of MF samples, a robust region growing algorithm is employed. For each seed pixel of MF class $i$ obtained from Step 1, its unclassified adjacent pixels are examined using an adaptive distance classifier augmented with spatial connectivity. A pixel is classified into class $i$ if the distance of the pixel to this class is within a threshold. The added pixel then becomes a new seed whose neighbours are continually inspected for inclusion in the region.

The distance of the pixel $d_{i}$ to class $i$ is calculated based on two factors: the intensity similarity of the pixel to the class mean, and the number of neighbouring pixels in the current slice belonging to this class,

$$
d_{i}=\frac{\left(I-\mu_{i}\right)^{2}}{2 \sigma^{2}}+\alpha *\left|I-\mu_{i}\right| * n
$$

where $I$ denotes the intensity of the observed pixel, $\mu_{i}$ is the mean intensity of the $i$ th class in this region based on current classification, and $\sigma$ is used to control the tolerance of intensity deviation. The weight of neighbourhood constraint is defined by a negative value of $\alpha$, and $n$ indicates the number of adjacent pixels in the current slice belonging to the $i$ th class.

The first term aims to recruit miss-detected pixels from the global thresholding by inclusion of small, local inhomogeneity. The second term aims to include more candidates from image noise by imposing spatial continuity. Therefore, the proposed adaptive distance classifier helps to handle intra-slice inhomogeneity and encompass outliers into a correct class. Using the adaptive distance classifier iteratively until the change in number of pixels is below a threshold, enabled the accurate development of smoothly connected MF regions, as shown in Fig. 1(c). After most of the MF regions are determined, an initial estimation of MB is obtained as the remaining pixels in the thigh area.

\subsection{Polynomial based 3D intensity inhomogeneity modelling}

Using the above described adaptive distance classifier and region-growing algorithm did not always identify all of the adipose tissue, mainly due to unexpected intra-slice intensity inhomogeneity and difficulty reaching all regions within the image. To fully address inhomogeneity in a MRI sequence, we propose a 3D polynomial method which is capable of simultaneously modelling both intraand inter-slice inhomogeneity and at a significant level. In contrast with most previous methods, which account only for intra-slice intensity variations [5], the proposed method produces a uniform, concise parametric representation and 
smooth estimation in 3D, which is superior to simply using a stack of separately fitted polynomial surfaces.

To generate the mapping function between the pixel locations and intensity values for each class, a least-square high-order polynomial fitting is employed. We assume that the intensity of a pixel $i$ at $\left(x_{i}, y_{i}, z_{i}\right)$ is represented by

$$
I\left(x_{i}, y_{i}, z_{i}\right)=\sum_{n=1}^{N} w_{n} F_{n}\left(x_{i}, y_{i}, z_{i}\right)+\eta\left(x_{i}, y_{i}, z_{i}\right)
$$

where $F_{n}$ represents basis functions, $N$ is the total number of basis functions, and $w_{n}$ denotes the weights that need to be determined, $\eta$ represents the intensity error at this pixel.

The basis functions used in our study are in the form of

$$
F_{n}(x, y, z)=x^{p} y^{q} z^{r}
$$

where $0 \leq p, q, r \leq k$ and $p+q+r \leq k, k$ is the degree of the chosen polynomial. In our study, polynomials of degree between 4 to 6 are experimentally shown to give the best results.

Let the segmented pixels of a particular class obtained from the initial segmentation be $\left\{x_{i}, y_{i}, z_{i} \mid 1 \leq i \leq m\right\}$. The total squared error can be calculated by

$$
\epsilon^{2}=\sum_{i=1}^{m}\left(I\left(x_{i}, y_{i}, z_{i}\right)-\sum_{n=1}^{N} w_{n} F_{n}\left(x_{i}, y_{i}, z_{i}\right)\right)^{2}
$$

The weighting coefficients $w_{n}$ can be determined by solving the least mean square problem using standard regression techniques.

In terms of the sample set of each class for polynomial fitting, MF-MB candidates have been obtained from the initial estimation. They are distributed thoroughly across the whole MRI sequence, holding sufficient information about spacial intensity variation. However, they may contain erroneous pixels due to over-segmentation in largely corrupted MRI. To obtain reliable sample sets, we generate the histograms of classified pixels for both classes separately, and examine if there is any overlap of non-zero bins between them. A bin with overlap may contain ambiguous segmentation. To remove this ambiguity, we first determine the midpoint value of overlapping bin(s). We then reject all the MF pixels with intensity below this midpoint, and reject all the MB pixels with intensity above the midpoint. This approach ensures that any segmented points with ambiguity from initial estimation will not be used in polynomial fitting, thereby improving accuracy on intensity inhomogeneity mapping.

\subsection{Segmentation refinement}

After the adaptive distance classifier based region growing, classification of most MF pixels can be obtained. However, the segmentation is not yet complete. A considerable number of fat pixels may remain undetected in regions with excessive intensity variations or in regions not yet reached by region growing. 
Classified pixels residing in overlapping bins are ambiguous and may be erroneous. To recruit undetected MF pixels and refine the obtained classification, inhomogeneity maps generated as described above are used.

For each unclassified or ambiguous pixel, an absolute difference between the actual pixel intensity $I(x, y, z)$ and the predicted value from each inhomogeneity map at location $(x, y, z)$ is calculated. This gives a score indicating the probability of a pixel belonging to either class, and thus classifying the pixel. The example of complete segmentation using the 3D IIM is shown in Fig. 1(e). It is clearly seen that MF pixels and small regions missed out by a global thresholding or not reachable by adaptive region growing are successfully identified.

\section{Experimental Results}

The proposed MB-MF segmentation method has been successfully applied to analyse a series of thigh MR images. In this section, we demonstrate the effectiveness of our method through the example of a representative thigh MRI scan obtained from an elderly man. The scan generated 24 serial-slices. The images were corrupted by noise and exhibited a significant amount of intensity inhomogeneity. Ordinary automated segmentation methods cannot succeed in such MRIs Using the proposed auto-segmentation method, the MF-MB segmentation results are presented in Fig. 2. Good classification results have been achieved on different tissue types, including for the first time, quantification of unconnected and highly irregular fat tissues.
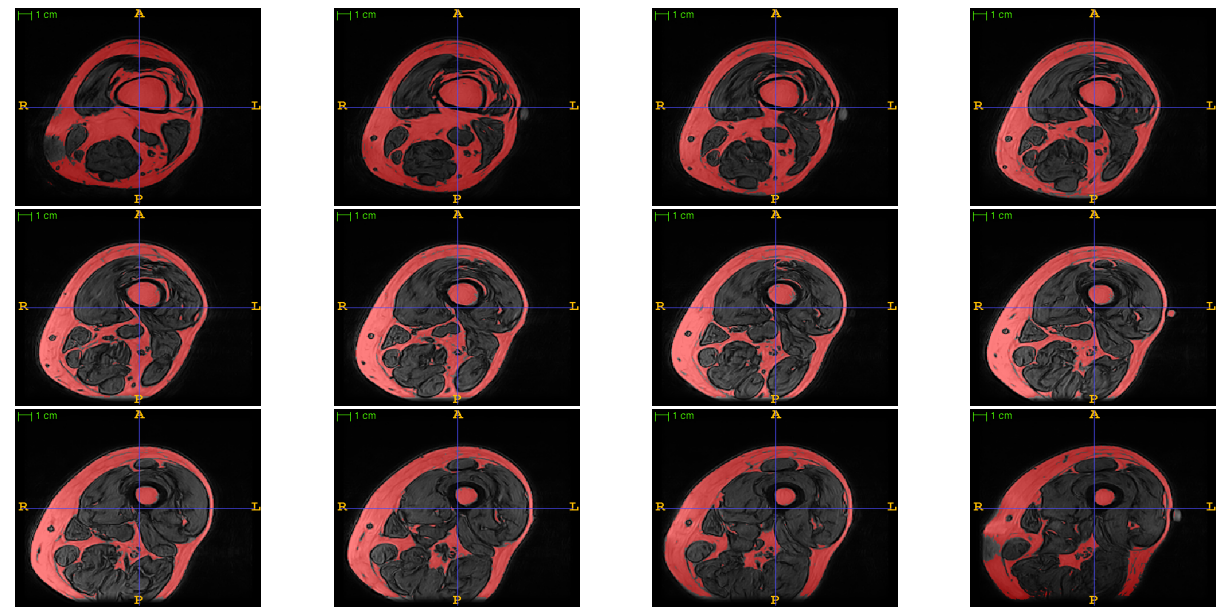

Fig. 2. MF-MB segmentation on an old person with excessive fat tissue. Results show every odd slice from the 1st to $23 \mathrm{rd}$ in the thigh MRI scan.

To quantitatively evaluate the effectiveness of the method, we compared the segmentation accuracy with professionally approved hand-labelled benchmarks. 
Figure 3 shows the accuracy of using robust region growing (RRG) augmented with spatial continuity, and the accuracy of also employing intensity inhomogeneity mapping. The results show that an average accuracy of $86 \%$ over the whole scan was achieved by robust region growing, and a further improvement to $92 \%$ average accuracy was made by employing the 3D intensity inhomogeneity mapping. The accuracy average was calculated based on all 24-slices, including the beginning and the last few slices, which normally exhibit extreme image noise and intensity inhomogeneity. As shown in Fig. 3, the average percentage of adipose MF over the thigh area is around $38 \%$.

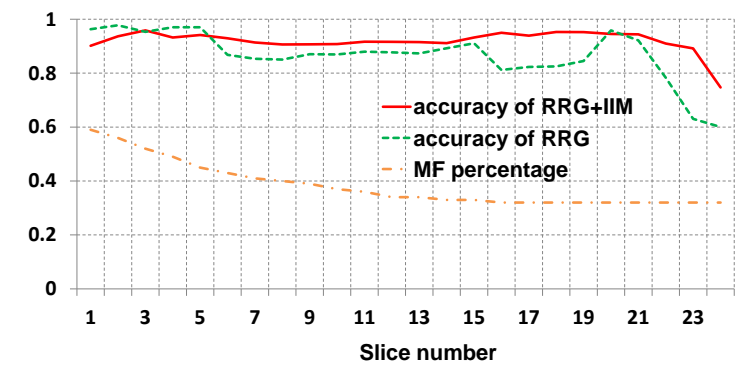

Fig. 3. MF percentage and segmentation accuracy when using robust region growing (RRG) and RRG combined with IIM.

Intensity variation and overlap between the two classes are shown in Fig. 4. We observe a remarkable level $25 \sim 30 \%$ of the intensity overlapping between the two classes in each slice. Meanwhile, the mean intensity value of each class varies significantly throughout the scan. For example, in the 12 bit grayscale MRI of a total 4096 intensity values, there is a variation of around 2000 values for $\mathrm{MF}$ and 600 values for MB.

The proposed hybrid automatic MRI segmentation method is conceptually simple and straightforward to implement. The algorithm was implemented in MATLAB and tested on a PC with a dual-core $2.5 \mathrm{GHz}$ processor and 4GB RAM. The time taken to process the whole 24 slices at the stage of robust region growing was around 100 seconds, and the additional time used for generating the $3 \mathrm{D}$ intensity maps and segmentation refinement was only around 25 seconds. Compared with the many hours of time spent on manual segmentation (for example using ITK-SNAP [2]) the proposed auto-segmentation method presents a significant advantage.

\section{Conclusion}

A novel approach has been presented for segmenting a large amount of complex adipose tissues from thigh MRIs. The proposed method is fully automatic. It does not require any manual interaction or training examples pre-generated by 


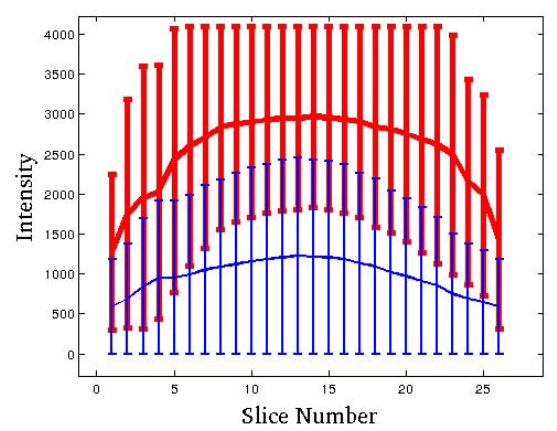

Fig. 4. Intensity range of $\mathrm{MF}$ and $\mathrm{MB}$ class is shown by vertical red and blue lines respectively; mean values are presented in corresponding colors using solid lines.

time-consuming hand-labelling. The accuracy and performance has been demonstrated by the experimental results in the presence of a significant amount of intensity inhomogeneity and image noise. Based on the MB-MF segmentation, marrow can then be easily distinguished from fat tissues, since it is well encompassed by the strongly contrasting bone. The method has proven to be effective in drastically speeding up the process of quantifying fat tissues in our research project into ageing.

\section{References}

1. Sharma, N., Aggarwal, L.: Automated medical image segmentation techniques. J. Med. Phys. 35(1) (2010) 3-14

2. Yushkevich, P.A., Piven, J., Cody Hazlett, H., Gimpel Smith, R., Ho, S., Gee, J.C., Gerig, G.: User-guided 3D active contour segmentation of anatomical structures: Significantly improved efficiency and reliability. Neuroimage 31(3) (2006) 1116-1128

3. McPhee, J., Williams, A., Stewart, C., Baar, K., Schindler, J.: The training stimulus experienced by the leg muscles during cycling in humans. Exp Physiol 94(684-694) (2009) 6

4. Chuang, K., Tzeng, H., Chen, S., Wu, J., Chen, T.: Fuzzy C-means clustering with spatial information for image segmentation. Computerized Medical Imaging and Graphics 30(1) (2006) 9-15

5. Vovk, U., Pernus, F., Likar, B.: A review of methods for correction of intensity inhomogeneity in MRI. IEEE Trans. Medical Imaging 26(3) (2007) 405-421

6. Dawant, B., Zijdenbos, A., Margolin, R.: Correction of intensity variations in MR images for computer-aided tissue classification. IEEE Trans. Medical Imaging 12(4) (1993) 770-781

7. Meyer, C., Bland, P., Pipe, J.: Retrospective correction of intensity inhomogeneities in MRI. IEEE Trans. Medical Imaging 14(1) (1995) 36-41 